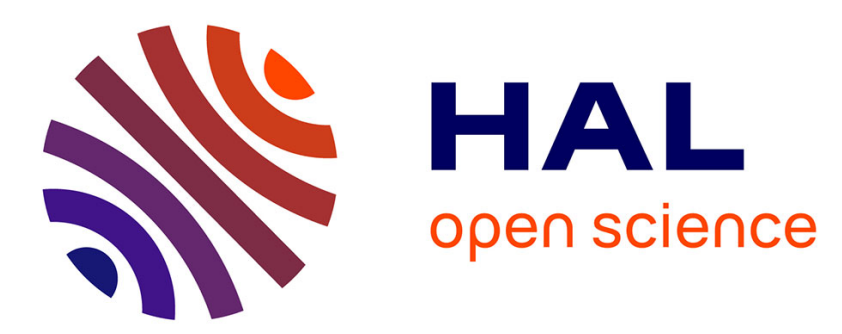

\title{
The effect of substrate structure on the chemoselectivity of Candida antarctica lipase B-catalyzed acylation of amino-alcohols
}

Florian Le Joubioux, Yesmine Ben Henda, Nicolas Bridiau, Oussama Achour, Marianne Graber, Thierry Maugard

\section{- To cite this version:}

Florian Le Joubioux, Yesmine Ben Henda, Nicolas Bridiau, Oussama Achour, Marianne Graber, et al.. The effect of substrate structure on the chemoselectivity of Candida antarctica lipase B-catalyzed acylation of amino-alcohols. Journal of Molecular Catalysis B: Enzymatic, 2012, 85-86, pp.193-199. hal-00789681

\section{HAL Id: hal-00789681 \\ https://hal.science/hal-00789681}

Submitted on 18 Feb 2013

HAL is a multi-disciplinary open access archive for the deposit and dissemination of scientific research documents, whether they are published or not. The documents may come from teaching and research institutions in France or abroad, or from public or private research centers.
L'archive ouverte pluridisciplinaire HAL, est destinée au dépôt et à la diffusion de documents scientifiques de niveau recherche, publiés ou non, émanant des établissements d'enseignement et de recherche français ou étrangers, des laboratoires publics ou privés. 
1 The effect of substrate structure on the chemoselectivity of Candida

2 antarctica lipase B-catalyzed acylation of amino-alcohols

3 Florian Le Joubioux, Yesmine Ben Henda, Nicolas Bridiau, Oussama Achour, Marianne

4 Graber, Thierry Maugard*

5

6 UMR 7266 CNRS-ULR, LIENSs, Equipe Approches Moléculaires, Environnement-Santé,

7 Université de La Rochelle, Avenue Michel Crépeau, 17042 La Rochelle, France.

8

9

10 *Author for correspondence (Fax: +33 546458277; E-mail: tmaugard@ univ-lr.fr)

11 
13 The selective acylation of multifunctional compounds exhibiting both alcohol and amino 14 groups gives interesting products with many applications in food, cosmetic and pharmaceutical industries, but it is real challenge. The current work describes the different behavior shown by Candida antarctica lipase B (Novozym 435) when catalyzing the O17 acylation and $\mathrm{N}$-acylation of bifunctional acyl acceptors. The acylation of three amino18 alcohols (alaninol, 4-amino-1-pentanol and 6-amino-1-hexanol) was studied using myristic acid as an acyl donor. To achieve this, a structure-reactivity study was performed in tert-amyl alcohol as a solvent, comparing the three amino-alcohols as acyl acceptors and a series of structurally related amines, namely (R)-sec-butylamine, 1-methoxy-2-propylamine and 1,2diaminopropane. These substrates were designed to investigate the effect of the group located in $\beta$-position of the amino group on the acyl acceptor: the more nucleophilic the group, the more the apparent maximal velocity $\left(V_{\text {max,app }}\right)$ of $\mathrm{N}$-acylation increases. Moreover, the crucial role of the carbon chain length between the alcohol and amino groups on the chemoselectivity was also demonstrated. The chemoselectivity for the $\mathrm{N}$-acylation was improved when the carbon chain included two carbons (alaninol) whereas the chemoselectivity for the Oacylation was improved when the carbon chain included four carbons or more (4-amino-1pentanol and 6-amino-1-hexanol).

These results provided new insights for the selective synthesis of amides or esters produced

31 from the acylation of bifunctional substrates.

32 Keywords: N-acylation; O-acylation; Candida antarctica lipase B; Organic solvent; 33 Chemoselectivity 


\section{Introduction}

The selective acylation of amino-alcohols have applications in many areas, such as pharmaceutics and cosmetics, through the synthesis of ceramides $[1,2]$ as potential anti-viral or anti-tumor drugs [3, 4] and anti-oxidant stabilizers [5], or for the environmental, food, and agricultural industries, through the synthesis of glucamide or amino-acid based surfactants [68].

Chemical acylation of amino-alcohols is well established but the methods used are faced with several limitations. They need fastidious steps of alcohol group protection and deprotection for the control of chemoselectivity and stereoselectivity. The high temperatures often required for chemical synthesis also preclude the use of fragile molecules and may cause coloration of final products. In addition, the coproduction of salts and the use of toxic solvents (dimethylformamide, methanol, ...) that must be eliminated at the end of the reaction increase the cost of the processes. The use of biocatalysts can be an interesting alternative, which offers a clean way to perform chemical processes under mild reaction conditions and with a high degree of selectivity. The use of immobilized enzymes in organic media, in particular lipases (E.C. 3.1.1.3) provides several advantages such as shifting of the thermodynamic equilibrium in favor of synthesis over hydrolysis reaction, increasing solubility of non-polar substrates, eliminating side reactions, making easier enzyme recovery and increasing enzyme thermostability. Lipases are the most used enzymes for organic synthesis. They have been used to catalyze $\mathrm{O}$-acylation, transesterification and $\mathrm{N}$-acylation reactions to synthesize various multifunctional derivates related to pharmaceuticals, cosmetics and foods. However, despite the large amount of studies on key enzyme properties in biocatalysis, their chemoselectivity is still not completely understood. It is therefore still necessary to optimize the output of lipase-catalyzed reactions and make efforts to understand lipase chemoselectivity. 
Despite the attractive properties of lipases in organic solvents, few studies have been devoted to the lipase-catalyzed acylation of bifunctional molecules exhibiting both amino and alcohol groups such as ethanolamine, diethanolamine, 2-amino-1-butanol, 6-amino-1-hexanol, serine and amino-alcohols with variable carbon chain length [9-14]. In such reactions, the lipase was seen to catalyze $\mathrm{O}$-acylation or $\mathrm{N}$-acylation, with a chemoselectivity which is largely dependent on amino-alcohol structure.

Among lipases used in organic synthesis, Candida antarctica lipase B is well known for its ability to convert alcohols and amines into esters and amides in various organic solvents [8, 15] and seems to be the ideal enzyme for the acylation of compounds such as amino-alcohols. In the present work, we thus investigated the Candida antarctica lipase B-catalyzed acylation of various amines and amino-alcohols as acyl acceptors, using myristic acid as an acyl donor. The results obtained under a kinetic approach were analyzed by comparing the apparent kinetic parameters $V_{\mathrm{max}, a p p}$ and $K_{\mathrm{m} \text {,app }}$ obtained for each acyl acceptor.

\section{Materials and Methods}

\subsection{Enzyme and chemicals}

Novozym $^{\circledR} 435$ (immobilized Candida antarctica lipase B), was kindly provided by Novozymes A/S, Bagsvaerd, Denmark. ( \pm )-alaninol 1 (98\%), (R)-sec-butylamine 3 (99\%), ( \pm )-1-methoxy-2-propylamine 5 (95\%), ( \pm )-1,2-diaminopropane $7(\geq 98 \%)$ and 6-amino-1hexanol 11 ( $\geq 97 \%)$, as well as tert-amyl alcohol (99\%) were purchased from Sigma-Aldrich (St Louis, USA) while ( \pm )-4-amino-1-pentanol 9 was from Santa Cruz Biotechnology (USA). Myristic acid and acetic acid were from Fluka (St Quentin-Fallavier, Switzerland). All chemicals were dried over molecular sieves. Pure water was obtained via a Milli-Q system (Millipore, France). Acetonitrile and methanol were purchased from Carlo ERBA (Val-deReuil, France). 


\subsection{Enzymatic reactions}

In all cases, reactions were carried out in tert-amyl alcohol at $55^{\circ} \mathrm{C}$ in screw-caped tubes. $2 \mathrm{ml}$ reaction mixtures containing various amounts of acyl acceptor substrates (25-350 mM) and $175 \mathrm{mM}$ of myristic acid as an acyl donor were incubated for 10 minutes prior to addition of $50 \mathrm{~g} \mathrm{l}^{-1}$ of Candida antarctica lipase B for the acylation of sec-butylamine 3 or $5 \mathrm{~g} \mathrm{l}^{-1}$ of Candida antarctica lipase B for the acylation of another acyl acceptor. $100 \mu 1$ samples were taken at intervals and centrifuged at $14,000 \mathrm{rpm}$. The supernatants were then analyzed by LCMS, leading to the determination and quantification of remaining substrates and synthesized products. Initial rate measurements were performed according to a previously established procedure [16]. The initial rates were calculated from the linear relationship of the total concentration of products against reaction time $(0-2 \mathrm{~h})$.

\subsection{Evaluation of the chemoselectivity}

The chemoselectivity of Candida antarctica lipase B during the acylation of amino-alcohols was studied by comparing the alcohol group O-acylation and the amino group $\mathrm{N}$-acylation, and then calculated via the apparent catalytic efficiency ratio (Eq. (1)) [17], which was transformed into an apparent maximal velocity ratio (Eq. (2)) owing to the fact that $K_{\mathrm{m} \text {,app }}$ were identical for a given amino-alcohol.

$C=\left(V_{\text {max,app O-acylation }} / K_{\mathrm{m}, \text { app }}\right) /\left(V_{\text {max,app N-acylation }} / K_{\mathrm{m}, \text { app }}\right)(1)$

$C=V_{\text {max,app O-acylation }} / V_{\text {max,app N-acylation }}(2)$

\subsection{HPLC and structural analysis}

Structural and quantitative analysis of reaction products were conducted using a LC/MS-ES system from Agilent (1100 LC/MSD Trap mass spectrometer VL) with a C18 Prontosil 1205-C18-AQ reversed-phase column $(250 \times 4 \mathrm{~mm}, 5 \mu \mathrm{m}$; Bischoff Chromatography). Products were detected and quantified by differential refractometry and UV detection at $210 \mathrm{~nm}$. An 
external calibration was performed with pure myristic acid. Then calibrations for individual

109 acylation products were obtained after mass balance in reaction conditions enabling to obtain 110 only these products with myristic acid as an acyl donor. Low-resolution mass spectral

111 analyses were obtained by electrospray in the positive detection mode. Nitrogen was used as

112 the drying gas at $151 \mathrm{~min}^{-1}$ and $350{ }^{\circ} \mathrm{C}$ at a nebulizer pressure of 4 bars. The scan range was $11350-1000 \mathrm{~m} / \mathrm{z}$ using five averages and $13,000 \mathrm{~m} / \mathrm{z}$ per second resolution. The capillary voltage

114 was 4000 V. Processing was done offline using HP Chemstation software.

115 Various eluent systems were used depending on the acyl acceptor used. Reaction samples

116 resulting from the acylation of (R)-sec-butylamine 3 were eluted with acetonitrile/water/acetic

117 acid $(90 / 10 / 0.1, \mathrm{v} / \mathrm{v} / \mathrm{v})$ at room temperature and at a flow rate of $1 \mathrm{ml} \mathrm{min}^{-1}$. Reaction samples

118 resulting from the acylation of 1-methoxy-2-propylamine 5 and 1,2-diaminopropane 7 were

119 eluted with methanol/water/acetic acid (95/5/0.1 and 93/7/0.1, v/v/v, respectively) at room 120 temperature and at a flow rate of $1 \mathrm{ml} \mathrm{min}^{-1}$. The elution of reaction samples resulting from

121 the acylation of amino-alcohols1, 9 and11, was carried out at room temperature and at a flow

122 rate of $1 \mathrm{ml} \mathrm{min}^{-1}$, using a gradient that was derived from two eluent mixtures (Table 1).

124 Table 1. Elution gradient for HPLC analysis of reaction samples resulting from the acylation 125 of amino-alcohols $\mathbf{1 , 9}$ and $\mathbf{1 1 .}$

\begin{tabular}{|c|c|c|}
\hline $\begin{array}{c}\text { Time } \\
(\mathrm{min})\end{array}$ & $\begin{array}{c}\text { Solvent A: } \\
\text { acetonitrile/water/acetic } \\
\text { acid }(77 / 23 / 0.1, \mathrm{v} / \mathrm{v} / \mathrm{v}) \\
(\%)\end{array}$ & $\begin{array}{c}\text { Solvent B: } \\
\text { methanol/acetic acid } \\
(100 / 0.1, \mathrm{v} / \mathrm{v}) \\
(\%)\end{array}$ \\
\hline 0 & 100 & 0 \\
\hline 20 & 100 & 0 \\
\hline 23 & 0 & 100 \\
\hline 80 & 0 & 0 \\
\hline 82 & 100 & 0 \\
\hline 90 & 100 & 00 \\
\hline
\end{tabular}


In order to perform the purification and characterization of acylated products, $20 \mathrm{ml}$ reaction mixtures containing $50 \mathrm{mM}$ of the acyl acceptor and $175 \mathrm{mM}$ of myristic acid in tert-amyl alcohol were incubated for $24 \mathrm{~h}$ in presence of $15 \mathrm{~g} \mathrm{l}^{-1}$ of Candida antarctica lipase B. Purified products were then characterized by ${ }^{1} \mathrm{H}$ NMR and IR via preparative HPLC using a ProntoPrep C18 reversed-phase column $(250 \times 20 \mathrm{~mm}, 10 \mu \mathrm{m}$; Bischoff Chromatography) eluted with the gradient given in Table 1, at room temperature and at a flow rate of $5 \mathrm{ml} \mathrm{min}{ }^{-1}$. ${ }^{1} \mathrm{H}$ NMR were recorded on a JEOL-JNM LA400 spectrometer (400 MHz), with tetramethylsilane as an internal reference. Samples were studied as solutions in $\mathrm{CDCl}_{3}$. Infrared (IR) spectra were recorded from 400 to $4000 \mathrm{~cm}^{-1}$ with a resolution of $4 \mathrm{~cm}^{-1}$ using a 100 ATR spectrometer (Perkin-Elmer, United States).

N-myristyl 2-amino-1-propanol 2a: $\mathrm{m} / \mathrm{Z}\left(\mathrm{LR}-\mathrm{ESI}^{+}\right) \mathrm{C}_{17} \mathrm{H}_{35} \mathrm{NO}_{2}\left(\mathrm{M}+\mathrm{H}^{+}\right)$, found: 286.4, calculated for: 286.48. IR $v$ max $\left(\mathrm{cm}^{-1}\right)$ : 3100-3500 (O-H, alcohol and N-H, amide), 2800-3000 (CH of myristyl chain), 1638 (C=O, amide), 1543 (N-H, amide). ${ }^{1} \mathrm{H}$ NMR (400 $\left.\mathrm{MHz}, \mathrm{CDCl}_{3}, \delta \mathrm{ppm}\right): \delta 0.88\left(\mathrm{t}, 3 \mathrm{H}, J=6.06 \mathrm{~Hz},-\mathrm{CH}_{2}-\mathrm{C}_{3}\right), 1.17(\mathrm{~d}, 3 \mathrm{H}, J=6.06 \mathrm{~Hz},-\mathrm{CH}-$ $\left.\mathrm{C}_{3}\right), 1.25$ (m, 20H, - $\mathrm{CH}_{2^{-}}$of myristyl chain), 1.63 (m, $2 \mathrm{H},-\underline{\mathrm{C}}_{2}-\mathrm{CH}_{2}-\mathrm{CO}-\mathrm{NH}-$ of myristyl chain), 2.19 (t, $2 \mathrm{H}, J=6,06 \mathrm{~Hz},-\mathrm{CH}_{2}-\mathrm{C}_{2}-\mathrm{CO}-\mathrm{NH}$ - of myristyl chain), 3.04 (s, 1H, -OH), 3.27 $\left(\mathrm{dd}, 1 \mathrm{H}, \quad J=5 \mathrm{~Hz}, J=10 \mathrm{~Hz},-\mathrm{CH}-\underline{\mathrm{H}}_{2}-\mathrm{OH}\right), 3.46\left(\mathrm{dd}, 1 \mathrm{H}, J=3.7 \mathrm{~Hz}, J=11 \mathrm{~Hz},-\mathrm{CH}-\underline{\mathrm{C}}_{\underline{2}^{-}}\right.$ $\mathrm{OH}), 4.07$ (m, 1H, -CH-), 5.7 (s, 1H, -NH-).

N,O-dimyristyl 2-amino-1-propanol 2c: m/Z (LR-ESI $\left.{ }^{+}\right) \mathrm{C}_{31} \mathrm{H}_{62} \mathrm{NO}_{3}\left(\mathrm{M}+\mathrm{Na}^{+}\right)$, found: 518.6, calculated for: 518.85. IR $v$ max $\left(\mathrm{cm}^{-1}\right): 3301(\mathrm{~N}-\mathrm{H}$, amide), 2800-3000 (CH of myristyl chain), 1737 ( $\mathrm{C}=\mathrm{O}$, ester), 1643 ( $\mathrm{C}=\mathrm{O}$, amide), 1542 (N-H, amide). ${ }^{1} \mathrm{H}$ NMR (400 MHz, $\left.\mathrm{CDCl}_{3}, \delta \mathrm{ppm}\right): \delta 0.88\left(\mathrm{t}, 6 \mathrm{H}, J=7.6 \mathrm{~Hz}, 2 \mathrm{x}-\mathrm{CH}_{2}-\mathrm{CH}_{3}\right), 1.16\left(\mathrm{~d}, 3 \mathrm{H}, J=7.6 \mathrm{~Hz},-\mathrm{CH}-\underline{\mathrm{H}}_{3}\right)$, 1.25 (m, 40H, $-\mathrm{CH}_{2}$ - of myristyl chain), 1.6 (m, 4H, 2x $-\mathrm{C}_{2}-\mathrm{CH}_{2}-\mathrm{CO}-$ of myristyl chain), $2.14\left(\mathrm{t}, 2 \mathrm{H}, J=7.2 \mathrm{~Hz},-\mathrm{CH}_{2}-\underline{\mathrm{CH}}_{2}-\mathrm{CO}-\mathrm{O}-\right.$ of myristyl chain), 2.32 (t, $2 \mathrm{H}, J=7.2 \mathrm{~Hz},-\mathrm{CH}_{2}-$ $\mathrm{C}_{2}-\mathrm{CO}-\mathrm{NH}-$ of myristyl chain), 4 (dd, $\left.1 \mathrm{H}, J=4.4 \mathrm{~Hz}, J=10.7 \mathrm{~Hz},-\mathrm{CH}-\underline{\mathrm{C}}_{2}-\mathrm{O}-\right), 4.13$ (dd, 
$\left.1521 \mathrm{H}, J=4.9 \mathrm{~Hz}, J=10 \mathrm{~Hz},-\mathrm{CH}-\underline{\mathrm{H}}_{2}-\mathrm{O}-\right), 4.29(\mathrm{~m}, 1 \mathrm{H},-\mathrm{CH}-), 5.54(\mathrm{~d}, 1 \mathrm{H}, J=7.3 \mathrm{~Hz}, \mathrm{C}-\mathrm{N} \underline{\mathrm{H}}-$ $\left.153 \mathrm{CH}_{2^{-}}\right)$.

154

155

156

157

158

159

160

161

162

163

164

165

166

167

168

169

170

171

172

173

174

175

N-myristyl sec-butylamine 4a: $\mathrm{m} / \mathrm{Z}\left(\right.$ LR-ESI $\left.{ }^{+}\right) \mathrm{C}_{18} \mathrm{H}_{37} \mathrm{NO}\left(\mathrm{M}+\mathrm{H}^{+}\right)$, found: 284.2 , calculated for: 284.51. ${ }^{1} \mathrm{H} \mathrm{NMR}\left(400 \mathrm{MHz}, \mathrm{CDCl}_{3}, \delta \mathrm{ppm}\right): \delta 0.89\left(\mathrm{~m}, 6 \mathrm{H}, 2 \mathrm{x}-\mathrm{CH}_{2}-\mathrm{CH}_{3}\right), 1.1$ (d, $\left.3 \mathrm{H}, J=7 \mathrm{~Hz},-\mathrm{CH}-\underline{\mathrm{H}}_{3}\right), 1.25\left(\mathrm{~m}, 20 \mathrm{H},-\mathrm{CH}_{2}-\right.$ of myristyl chain), $1.43\left(\mathrm{~m}, 2 \mathrm{H},-\mathrm{CH}-\underline{\mathrm{C}}_{2} \underline{2}^{-}\right.$ $\left.\mathrm{CH}_{3}\right), 1.6\left(\mathrm{~m}, 2 \mathrm{H},-\mathrm{CH}_{2}-\mathrm{CH}_{2}-\mathrm{CO}-\mathrm{NH}-\right.$ of myristyl chain), 2.12 (st, $2 \mathrm{H}, \mathrm{J}=7 \mathrm{~Hz},-\mathrm{CH}_{2}-\mathrm{C}_{2} \underline{2}^{-}$ CO-NH- of myristyl chain), 3.9 (st, $1 \mathrm{H}, J=6.8 \mathrm{~Hz},-\mathrm{CH}-$ ), 5.17 (s, $1 \mathrm{H},-\mathrm{NH}-$ ).

N-myristyl 1-methoxy-2-propylamine 6a: $\mathrm{m} / \mathrm{Z}\left(\mathrm{LR}-\mathrm{ESI}^{+}\right) \mathrm{C}_{18} \mathrm{H}_{37} \mathrm{NO}_{2}\left(\mathrm{M}+\mathrm{H}^{+}\right)$, found: 300.3, calculated for: 300.51. IR $v \max \left(\mathrm{cm}^{-1}\right): 3304(\mathrm{~N}-\mathrm{H}$, amide), 2800-3000 (CH of myristyl chain), 1634 (C=O, amide), 1544 (N-H, amide). ${ }^{1} \mathrm{H}$ NMR (400 MHz, $\mathrm{CDCl}_{3}, \delta$ ppm): $\delta 0.88\left(\mathrm{t}, 3 \mathrm{H}, J=6.79 \mathrm{~Hz},-\mathrm{CH}_{2}-\mathrm{C}_{3} \underline{3}_{3}\right), 1.17\left(\mathrm{~d}, 3 \mathrm{H}, J=6.17 \mathrm{~Hz},-\mathrm{CH}-\underline{\mathrm{H}}_{3}\right), 1.26(\mathrm{~m}, 20 \mathrm{H},-$ $\mathrm{CH}_{2}$ - of myristyl chain), 1.62 (m, 2H, $-\mathrm{C}_{2}-\mathrm{CH}_{2}-\mathrm{CO}-\mathrm{NH}$ - of myristyl chain), 2.15 (t, $2 \mathrm{H}, J=$ $6.8 \mathrm{~Hz},-\mathrm{CH}_{2}-\mathrm{CH}_{2}-\mathrm{CO}-\mathrm{NH}-$ of myristyl chain), $2.19\left(\mathrm{t}, 1 \mathrm{H}, J=6.8 \mathrm{~Hz},-\mathrm{CH}-\underline{\mathrm{H}}_{2}-\mathrm{OCH}_{3}\right), 3.36$ $\left(\mathrm{m}, 3 \mathrm{H},-\mathrm{OC} \underline{H}_{3}\right), 4.06\left(\mathrm{t}, 1 \mathrm{H}, J=6.45 \mathrm{~Hz},-\mathrm{CH}-\underline{\mathrm{C}}_{2}-\mathrm{OCH}_{3}\right), 4.16(\mathrm{~m}, 1 \mathrm{H},-\mathrm{CH}-), 5.62(\mathrm{~d}, 1 \mathrm{H}$, $J=5.84 \mathrm{~Hz},-\mathrm{NH}-)$.

1-N-myristyl 1,2-diaminopropane 8a: m/Z (LR-ESI $\left.{ }^{+}\right) \mathrm{C}_{17} \mathrm{H}_{36} \mathrm{~N}_{2} \mathrm{O}\left(\mathrm{M}+\mathrm{H}^{+}\right)$, found: 285.4, calculated for: $285.49 .{ }^{1} \mathrm{H} \mathrm{NMR}\left(400 \mathrm{MHz}, \mathrm{CDCl}_{3}, \delta \mathrm{ppm}\right): \delta 0.88(\mathrm{t}, 3 \mathrm{H}, J=6.67 \mathrm{~Hz},-$ $\left.\mathrm{CH}_{2}-\underline{\mathrm{C}}_{3}\right), 1.22\left(\mathrm{~d}, 3 \mathrm{H}, J=6.67 \mathrm{~Hz},-\mathrm{CH}-\underline{\mathrm{C}}_{3}\right), 1.25$ (m, $20 \mathrm{H},-\mathrm{CH}_{2^{-}}$of myristyl chain), 1.61 (q, $2 \mathrm{H}, J=6.67 \mathrm{~Hz},-\underline{\mathrm{C}}_{2}-\mathrm{CH}_{2}-\mathrm{CO}-\mathrm{NH}-$ of myristyl chain), $1.96\left(\mathrm{~s}, 2 \mathrm{H},-\mathrm{NH}_{2}\right), 2.19$ (t, $2 \mathrm{H}, J=$ 7.3Hz, $-\mathrm{CH}_{2}-\mathrm{CH}_{2}-\mathrm{CO}-\mathrm{NH}-$ of myristyl chain), 3.21(st, $1 \mathrm{H}, J=5.9 \mathrm{~Hz},-\mathrm{CH}-$ ), 3.27 (qd, $1 \mathrm{H}$, $\left.J=2.6 \mathrm{~Hz}, J=11.8 \mathrm{~Hz},-\mathrm{CH}-\underline{\mathrm{H}}_{2}-\mathrm{NH}-\right), 3.46\left(\mathrm{qd}, 1 \mathrm{H}, J=3.24 \mathrm{~Hz}, J=13.6 \mathrm{~Hz},-\mathrm{CH}-\underline{\mathrm{C}}_{2}-\mathrm{NH}-\right)$, $7.22(\mathrm{t}, 1 \mathrm{H}, J=4.42 \mathrm{~Hz},-\mathrm{NH}-)$.

N-myristyl 4-amino-1-pentanol 10a: $\mathrm{m} / \mathrm{Z}\left(\mathrm{LR}_{-\mathrm{ESI}}^{+}\right) \mathrm{C}_{19} \mathrm{H}_{39} \mathrm{NO}_{2}\left(\mathrm{M}+\mathrm{H}^{+}\right)$, found: 314.2 calculated for: 314.53 . IR $v$ max $\left(\mathrm{cm}^{-1}\right)$ : 3200-3500 (O-H, alcohol and N-H, amide), 
176

177

178

179

180

181

182

183

184

185

186

187

188

189

190

191

192

193

194

195

196

197

198

199

2800-3000 ( $\mathrm{CH}$ of myristyl chain), 1639 (C=O, amide), 1545 (N-H, amide). ${ }^{1} \mathrm{H}$ NMR (400 $\left.\mathrm{MHz}, \mathrm{CDCl}_{3}, \delta \mathrm{ppm}\right): \delta 0.88\left(\mathrm{t}, 3 \mathrm{H}, J=6.58 \mathrm{~Hz},-\mathrm{CH}_{2}-\mathrm{C}_{3}\right), 1.14(\mathrm{~d}, 3 \mathrm{H}, J=6.23 \mathrm{~Hz},-\mathrm{CH}-$ $\left.\mathrm{C}_{3} \underline{3}\right), 1.25$ (m, 20H, $-\mathrm{CH}_{2}$ - of myristyl chain), 1.53 (m, 4H, $\left.-\mathrm{CH}-\underline{\mathrm{C}}_{2}-\underline{\mathrm{C}}_{2}-\mathrm{CH}_{2}-\mathrm{OH}\right), 1.63$ (m, $2 \mathrm{H},-\underline{\mathrm{C}}_{2}-\mathrm{CH}_{2}-\mathrm{CO}-\mathrm{NH}-$ of myristyl chain), $2.14\left(\mathrm{t}, 2 \mathrm{H}, \mathrm{J}=7.27 \mathrm{~Hz},-\mathrm{CH}_{2}-\mathrm{C}_{2}-\mathrm{CO}-\mathrm{NH}-\right.$ of myristyl chain), 2.94 (s, $1 \mathrm{H},-\mathrm{OH}), 3.67\left(\mathrm{~m}, 2 \mathrm{H},-\mathrm{CH}_{2}-\mathrm{C}_{2}-\mathrm{OH}\right), 4.06(\mathrm{~m}, 1 \mathrm{H},-\mathrm{CH}-), 5.28$ $(\mathrm{s}, 1 \mathrm{H},-\mathrm{NH}-)$.

O-myristyl 4-amino-1-pentanol 10a: $\mathrm{m} / \mathrm{Z}\left(\mathrm{LR}_{-} \mathrm{ESI}^{+}\right) \mathrm{C}_{19} \mathrm{H}_{39} \mathrm{NO}_{2}\left(\mathrm{M}+\mathrm{H}^{+}\right)$, found: 314.2 calculated for: 314.53 . IR $v$ max $\left(\mathrm{cm}^{-1}\right): 3291(\mathrm{~N}-\mathrm{H}$, amine), 2800-3000 (CH of myristyl chain), 1736 (C=O, ester), $1557\left(\mathrm{~N}-\mathrm{H}\right.$, amine). ${ }^{1} \mathrm{H} \mathrm{NMR}\left(400 \mathrm{MHz}, \mathrm{CDCl}_{3}, \delta \mathrm{ppm}\right): \delta 0.88(\mathrm{t}$, $\left.3 \mathrm{H}, J=6.99 \mathrm{~Hz},-\mathrm{CH}_{2}-\underline{\mathrm{C}}_{3}\right), 1.14\left(\mathrm{~d}, 3 \mathrm{H}, J=8 \mathrm{~Hz},-\mathrm{CH}-\underline{\mathrm{C}}_{3}\right), 1.25\left(\mathrm{~m}, 20 \mathrm{H},-\mathrm{CH}_{2}\right.$ - of myristyl chain), 1.52 (m, 4H, - $\left.\mathrm{CH}-\underline{\mathrm{C}}_{2}-\underline{\mathrm{C}}_{2}-\mathrm{CH}_{2}-\mathrm{O}-\right), 1.62$ (m, 2H, $-\underline{\mathrm{C}}_{2}-\mathrm{CH}_{2}-\mathrm{CO}-\mathrm{O}-$ of myristyl chain), $2.22\left(\mathrm{t}, 1 \mathrm{H}, J=7.16 \mathrm{~Hz},-\mathrm{CH}_{2}-\underline{\mathrm{C}}_{2}-\mathrm{CO}-\mathrm{O}-\right.$ of myristyl chain), 2.29 (t, $1 \mathrm{H}, J=7.5 \mathrm{~Hz},-$ $\mathrm{CH}_{2}-\underline{\mathrm{C}}_{2}-\mathrm{CO}-\mathrm{O}$ - of myristyl chain), 3.43 (m, 1H, $-\mathrm{CH}_{2}-\underline{\mathrm{CH}}_{2}-\mathrm{O}-$ ), 3.69 (m, 1H, $-\mathrm{CH}_{2}-\underline{\mathrm{C}}_{2}-\mathrm{O}-$ ), 4.1 (m, 1H, -CH-).

N,O-dimyristyl 4-amino-1-pentanol 10c: $\mathrm{m} / \mathrm{Z}\left(\mathrm{LR}-\mathrm{ESI}^{+}\right) \mathrm{C}_{33} \mathrm{H}_{66} \mathrm{NO}_{3}\left(\mathrm{M}+\mathrm{Na}^{+}\right)$, found: 546.2 calculated for: 546.9. IR $v$ max $\left(\mathrm{cm}^{-1}\right): 3304(\mathrm{~N}-\mathrm{H}$, amide), 2800-3000 (CH of myristyl chain), 1732 ( $\mathrm{C}=\mathrm{O}$, ester), $1640\left(\mathrm{C}=\mathrm{O}\right.$, amide), $1546\left(\mathrm{~N}-\mathrm{H}\right.$, amide). ${ }^{1} \mathrm{H}$ NMR (400 $\left.\mathrm{MHz}, \mathrm{CDCl}_{3}, \delta \mathrm{ppm}\right): \delta 0.88\left(\mathrm{t}, 6 \mathrm{H}, J=7.43 \mathrm{~Hz}, 2 \mathrm{x}-\mathrm{CH}_{2}-\underline{\mathrm{C}}_{3}\right), 1.14(\mathrm{~d}, 3 \mathrm{H}, J=6.83 \mathrm{~Hz},-\mathrm{CH}-$ $\mathrm{C}_{3}$ ), 1.25 (m, $40 \mathrm{H},-\mathrm{CH}_{2}$ - of myristyl chain), 1.53 (m, 4H, $\left.-\mathrm{CH}-\underline{\mathrm{C}}_{2}-\mathrm{C}_{2}{ }_{2}-\mathrm{CH}_{2}-\mathrm{O}-\mathrm{C}\right), 1.6$ (m, 4H, 2x - $\underline{\mathrm{CH}}_{2}-\mathrm{CH}_{2}-\mathrm{CO}$ - of myristyl chain), 2.14 (t, $4 \mathrm{H}, J=7.08 \mathrm{~Hz},-\mathrm{CH}_{2}-\underline{\mathrm{C}}_{2}-\mathrm{CO}-$ of myristyl chain), 3.68 (m, 2H, $\left.-\mathrm{CH}_{2}-\underline{\mathrm{C}}_{2}-\mathrm{O}-\mathrm{C}\right), 4.07$ (m, $\left.1 \mathrm{H},-\mathrm{CH}-\right), 5.27$ (d, $1 \mathrm{H}, J=6.86 \mathrm{~Hz}$, $\mathrm{NH}-$ ).

N-myristyl aminohexanol 12a: $\mathrm{m} / \mathrm{Z}\left(\right.$ LR-ESI $\left.{ }^{+}\right) \mathrm{C}_{20} \mathrm{H}_{41} \mathrm{NO}_{2}\left(\mathrm{M}+\mathrm{H}^{+}\right)$, found: 329.5 calculated for: 328.56. IR $v$ max $\left(\mathrm{cm}^{-1}\right): 3385(\mathrm{O}-\mathrm{H}$, alcohol), $3314(\mathrm{~N}-\mathrm{H}$, amide), 2800-3000 
200 (CH of myristyl chain), 1634 (C=O, amide), 1534 (N-H, amide). ${ }^{1} \mathrm{H}$ NMR (400 MHz, $\mathrm{CDCl}_{3}$, $201 \delta \mathrm{ppm}): \delta 0.88\left(\mathrm{t}, 3 \mathrm{H}, J=7.5 \mathrm{~Hz},-\mathrm{CH}_{2}-\mathrm{C}_{3}\right), 1.25\left(\mathrm{~m}, 20 \mathrm{H},-\mathrm{CH}_{2}-\right.$ of myristyl chain), 1.51 (m, 202 $2 \mathrm{H},-\underline{\mathrm{C}}_{2}-\mathrm{CH}_{2}-\mathrm{CO}-\mathrm{O}-$ of myristyl chain), 1.59 (m, 4H, $\left.-\mathrm{CH}_{2}-\underline{\mathrm{C}}_{2}-\underline{\mathrm{C}}_{2}-\mathrm{CH}_{2}-\mathrm{OH}\right), 2.26$ (t, $2 \mathrm{H}$, 203 $J=7.65 \mathrm{~Hz},-\mathrm{CH}_{2}-\underline{\mathrm{C}}_{2}-\mathrm{CO}-\mathrm{OH}$ of myristyl chain), $2.72(\mathrm{~s}, 1 \mathrm{H},-\mathrm{OH}), 3.25(\mathrm{t}, 2 \mathrm{H}, J=7.07 \mathrm{~Hz}$, 204 $\left.-\mathrm{CH}_{2}-\mathrm{C}_{2}-\mathrm{OH}\right), 3.63$ (t, $\left.2 \mathrm{H}, J=7.29 \mathrm{~Hz},-\mathrm{CH}_{2}-\underline{\mathrm{C}}_{2}-\mathrm{NH}-\mathrm{CO}-\mathrm{CH}_{2}\right), 5.41$ (s, $1 \mathrm{H},-\mathrm{NH}-$ ).

O-myristyl aminohexanol 12b: $\mathrm{m} / \mathrm{Z}\left(\mathrm{LR}-\mathrm{ESI}^{+}\right) \mathrm{C}_{20} \mathrm{H}_{41} \mathrm{NO}_{2}\left(\mathrm{M}+\mathrm{H}^{+}\right)$, found: 329.5 206 calculated for: 328.56 . IR $v$ max $\left(\mathrm{cm}^{-1}\right): 3400(\mathrm{~N}-\mathrm{H}$, amine), 2800-3000 (CH of myristyl chain), 207 $1736\left(\mathrm{C}=\mathrm{O}\right.$, ester), $1544(\mathrm{~N}-\mathrm{H}$, amine $) .{ }^{1} \mathrm{H}$ NMR $\left(400 \mathrm{MHz}, \mathrm{CDCl}_{3}, \delta \mathrm{ppm}\right): \delta 0.88(\mathrm{t}, 3 \mathrm{H}, J=$ 208 7.28Hz, $\left.-\mathrm{CH}_{2}-\mathrm{CH}_{3}\right), 1.25$ (m, 20H, $-\mathrm{CH}_{2}$ - of myristyl chain), 1.55 (m, 2H, $-\mathrm{C}_{2}-\mathrm{CH}_{2}-\mathrm{CO}-\mathrm{O}-$ 209 of myristyl chain), $1.62\left(\mathrm{~m}, 4 \mathrm{H},-\mathrm{CH}_{2}-\underline{\mathrm{CH}}_{2}-\underline{\mathrm{C}}_{2}-\mathrm{CH}_{2}-\mathrm{NH}_{2}\right), 2.28\left(\mathrm{t}, 2 \mathrm{H}, J=7.65 \mathrm{~Hz},-\mathrm{CH}_{2}-\right.$ $\mathrm{C}_{2}-\mathrm{CO}-\mathrm{O}-$ of myristyl chain), 2.81 (s, $\left.2 \mathrm{H},-\mathrm{NH}_{2}\right), 3.64$ (t, $2 \mathrm{H}, J=6.47 \mathrm{~Hz},-\mathrm{CH}_{2}-\mathrm{C}_{2}-\mathrm{NH}_{2}$ ), $4.04\left(\mathrm{t}, 2 \mathrm{H}, \mathrm{J}=6.47 \mathrm{~Hz},-\mathrm{CH}_{2}-\mathrm{C}_{2}-\mathrm{O}-\mathrm{CO}-\mathrm{CH}_{2}\right)$.

N,O-dimyristyl aminohexanol 12c: $\mathrm{m} / \mathrm{Z}\left(\mathrm{LR}-\mathrm{ESI}^{+}\right) \mathrm{C}_{34} \mathrm{H}_{67} \mathrm{NO}_{3}\left(\mathrm{M}+\mathrm{Na}^{+}\right)$, found: 213 560.7, calculated for: 560.93. IR $v_{\max }\left(\mathrm{cm}^{-1}\right): 3298$ (N-H, amide), 2800-3000 (CH of myristyl chain), 1726 ( $\mathrm{C}=\mathrm{O}$, ester), 1635 ( $\mathrm{C}=\mathrm{O}$, amide), 1547 (N-H, amide). ${ }^{1} \mathrm{H}$ NMR (400 MHz, $\left.\mathrm{CDCl}_{3}, \delta \mathrm{ppm}\right): \delta 0.88\left(\mathrm{t}, 6 \mathrm{H}, J=6.48 \mathrm{~Hz}, 2 \mathrm{x}-\mathrm{CH}_{2}-\mathrm{C}_{3}\right), 1.25\left(\mathrm{~m}, 40 \mathrm{H},-\mathrm{CH}_{2}-\right.$ of myristyl chain), 1.5 (m, 4H, - $\underline{\mathrm{C}}_{2}-\mathrm{CH}_{2}-\mathrm{CO}$ - of myristyl chain), 1.6 (m, 4H, $-\mathrm{CH}_{2}-\underline{\mathrm{C}}_{2}-\mathrm{C}_{2}-\mathrm{CH}_{2}-\mathrm{O}-\mathrm{C}$ ), $2.15\left(\mathrm{t}, 2 \mathrm{H}, J=7.8 \mathrm{~Hz},-\mathrm{CH}_{2}-\underline{\mathrm{C}}_{2}-\mathrm{CO}-\mathrm{NH}-\right), 2.29$ (t, $\left.2 \mathrm{H}, J=7.8 \mathrm{~Hz},-\mathrm{CH}_{2}-\underline{C}_{2}-\mathrm{CO}-\mathrm{NH}-\right), 3.24$ $\left(\mathrm{q}, 2 \mathrm{H}, J=6.5 \mathrm{~Hz},-\mathrm{CH}_{2}-\underline{\mathrm{C}}_{2}-\mathrm{NH}-\right), 4.06\left(\mathrm{t}, 2 \mathrm{H}, J=5.9 \mathrm{~Hz},-\mathrm{CH}_{2}-\underline{\mathrm{H}}_{2}-\mathrm{O}-\mathrm{CO}-\mathrm{CH}_{2}\right), 5.4$ (s, $1 \mathrm{H},-\mathrm{NH}-)$.

\subsection{Analysis of the ionization state of substrates}

221 The ionization state of myristic acid in tert-amyl alcohol was investigated using infrared 222 spectroscopy analysis. Infrared (IR) spectra of samples containing $175 \mathrm{mM}$ myristic acid and 223 from 0 to $250 \mathrm{mM}$ alaninol were recorded from 1500 to $1800 \mathrm{~cm}^{-1}$ with a resolution of 4 
$224 \mathrm{~cm}^{-1}$ using a 100 ATR spectrometer (Perkin-Elmer, United States). Before the interpretation 225 of data, a treatment (base line correction, smoothing and normalization min-max) was applied 226 to spectra.

\section{Results and discussion}

228 In order to explore the chemoselectivity of the enzyme, kinetic studies were investigated for 229 the acylation of several acyl acceptors including various amines, methoxyamine and amino230 alcohols (Table 2), catalyzed by Candida antarctica lipase B using myristic acid (175 mM) 231 as an acyl donor and tert-amyl alcohol as solvent. From the LC-MS analysis, the decrease in 232 myristic acid concentration was always seen to be concomitant with the synthesis of acylated 233 products. The purification and the structural elucidation by mass spectroscopy, IR and NMR 234 analyses led to identify the structure of the acylated products described in Table 2. In absence 235 of enzyme, no product was detected within 2 days. 
Table 2. Structure of substrates and products resulting from the acylation of various acyl acceptors catalysed by Candida antarctica lipase B using myristic acid as an acyl donor in tert-amyl alcohol.

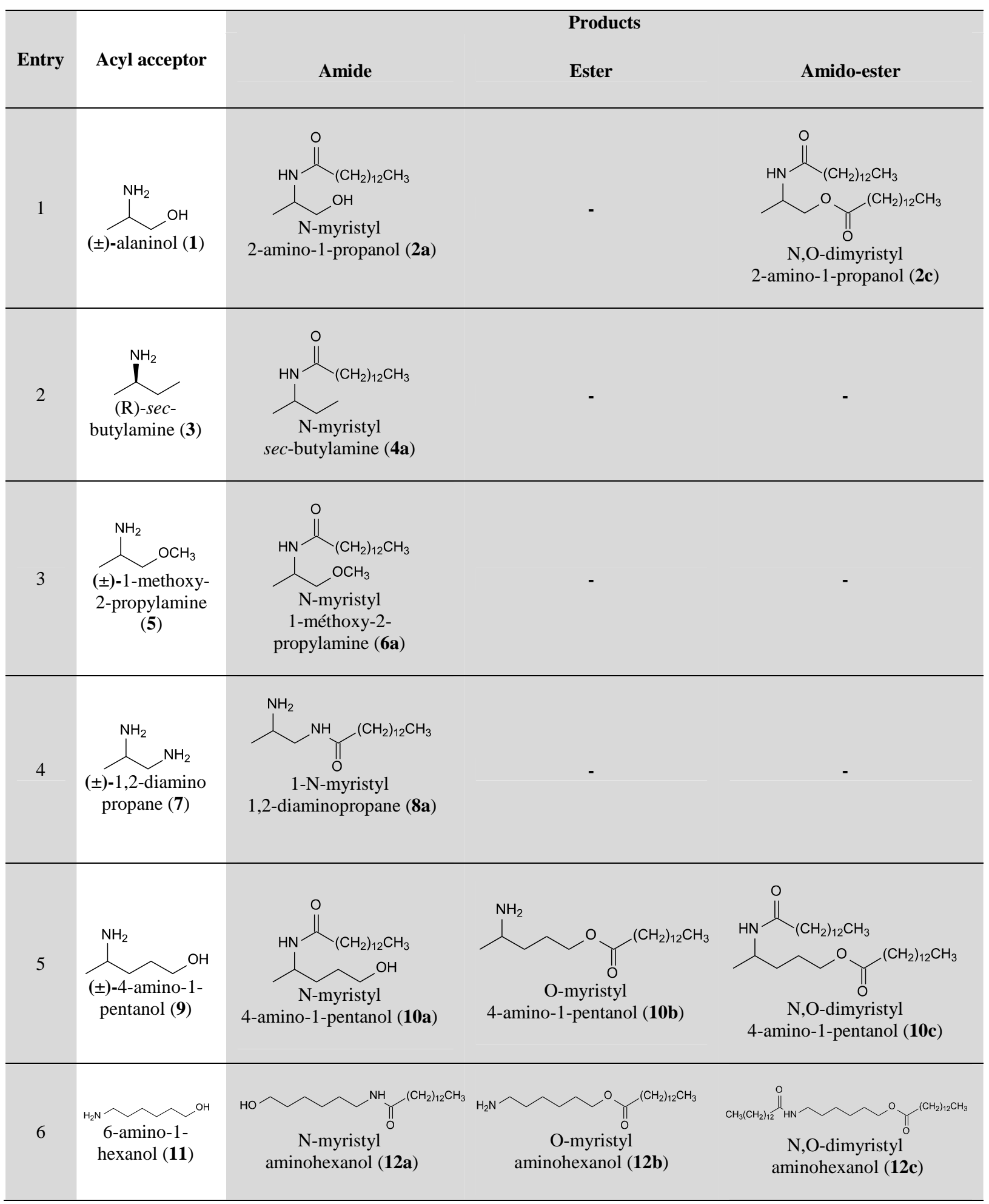


242 The acylation of alaninol (amino-alcohol 1, Table 2, entry 1) was chosen as the model 243 reaction to study the kinetic behaviour of Candida antarctica lipase B when catalyzing the 244 acylation of amino-alcohols. To achieve this, we determined the apparent kinetic parameters 245 of the amide and ester synthesis from myristic acid and alaninol. As no mono-O-acylation 246 product was detected under our experimental conditions, systematic analysis of the rates of 247 myristic acid conversion and alaninol $\mathrm{N}$-acylation were conducted, by varying alaninol 248 concentration. This analysis revealed Lineweaver-Burk reciprocal plots presentedon Fig. 1. 249 The intercepts of the y-axis and the x-axis gave the values of $V_{\text {max,app }}$ and $K_{\text {m,app }}$. The $V_{\max \text {,app }}$ 250 were found to be $4.9 \mathrm{mmol} \mathrm{h}^{-1} \mathrm{~g}^{-1}$ for myristic acid conversion and $4.3 \mathrm{mmol} \mathrm{h}^{-1} \mathrm{~g}^{-1}$ for 251 alaninol $\mathrm{N}$-acylation. The $V_{\text {max,app }}$ of $\mathrm{O}$-acylation of the $\mathrm{N}$-acylated product $2 \mathbf{a}$ was extremely 252 low $\left(0.3 \mathrm{mmol} \mathrm{h}^{-1} \mathrm{~g}^{-1}\right)$, which explained the similarities observed between the reciprocal rate 253 values of myristic acid conversion and alaninol $\mathrm{N}$-acylation. The $K_{\mathrm{m} \text {,app }}$ value of alaninol was 254 found to be $182 \mathrm{mM}$.

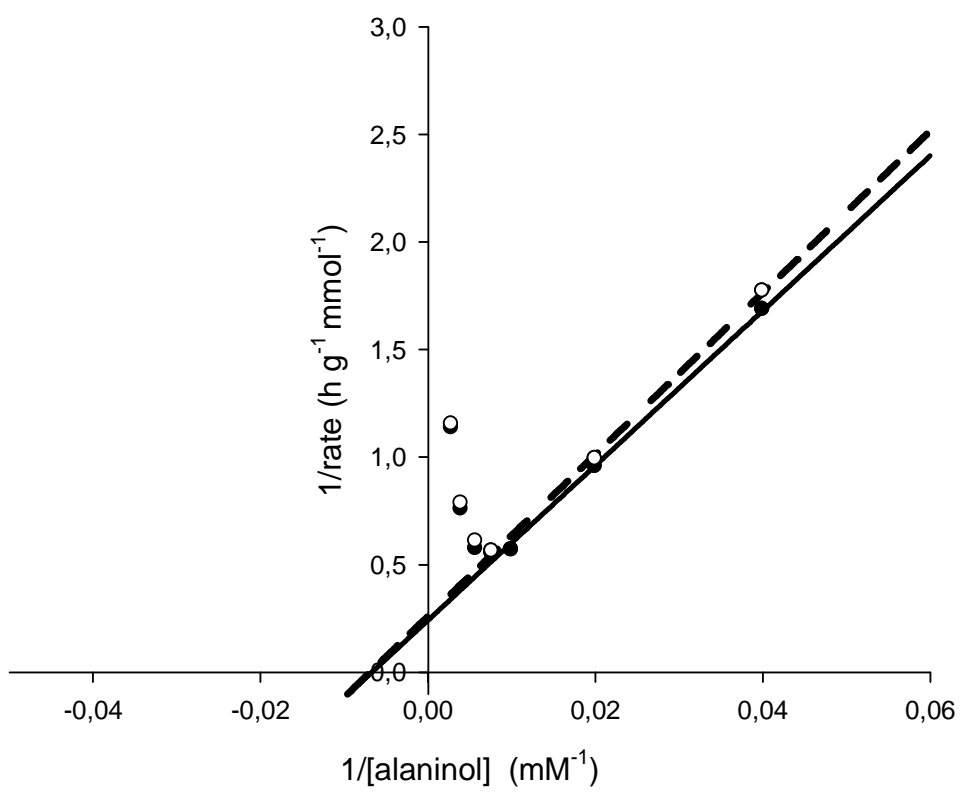

Fig. 1: Reciprocal initial rates of myristic acid conversion $(O)$ and alaninol $N$-acylation $(O)$ versus reciprocal alaninol concentrations. Reactions were carried out at $55^{\circ} \mathrm{C}$ using a fixed concentration of myristic acid $(175 \mathrm{mM})$ and $5 \mathrm{~g} \mathrm{l}^{-1}$ of Candida antarctica lipase B in $2 \mathrm{ml}$ of tert-amyl alcohol. The data represent the averages of triplicate runs whose standard deviations were always lower than $15 \%$. 
262 For high concentrations of amino-alcohol (superior to $100 \mathrm{mM}$ ), a decrease in initial rates was 263 observed. This phenomenon was most likely due to an inhibitor effect similar to an excess 264 substrate inhibition probably due to an interaction between myristic acid and the amino group 265 of alaninol. Indeed, the presence of an amino substrate and a fatty acid in an organic solvent 266 generally leads to the formation of an ion-pair complex between both substrates, depending 267 on the acido-basic conditions of the medium $[16,18,19]$. This salt complex makes the ion 268 forms of both substrates non reactive $\left(\mathrm{NH}_{3}{ }^{+}\right.$amine form and $\mathrm{COO}^{-}$fatty acid form) and 269 therefore leads to the overestimation of the substrate concentrations that are really available 270 for the enzyme in the reaction medium. This ion-pair complex was already described by 271 Maugard et al. [16] as a limiting factor of the lipase-catalyzed acylation under conditions 272 where it was less soluble than free substrates. To ascertain this hypothesis, the composition of 273 the medium, especially the carbonyl species, was analyzed by infrared spectroscopy at the 274 start of the reaction, for four concentrations of alaninol within the range 50-250 mM (Fig. 2). 275 When only myristic acid was solubilized in tert-amyl alcohol, only one carbonyl band was 276 observed at $1710 \mathrm{~cm}^{-1}$, corresponding to the acid form. When the concentration of alaninol 277 was increased, the carbonyl acid band disappeared in favor of a band at $1562 \mathrm{~cm}^{-1}$ 278 corresponding to a carboxylate ion. This additional band demonstrated the formation of an 279 ion-pair complex between myristic acid and alaninol when using an alaninol concentration 280 superior or equal to $100 \mathrm{mM}$. 


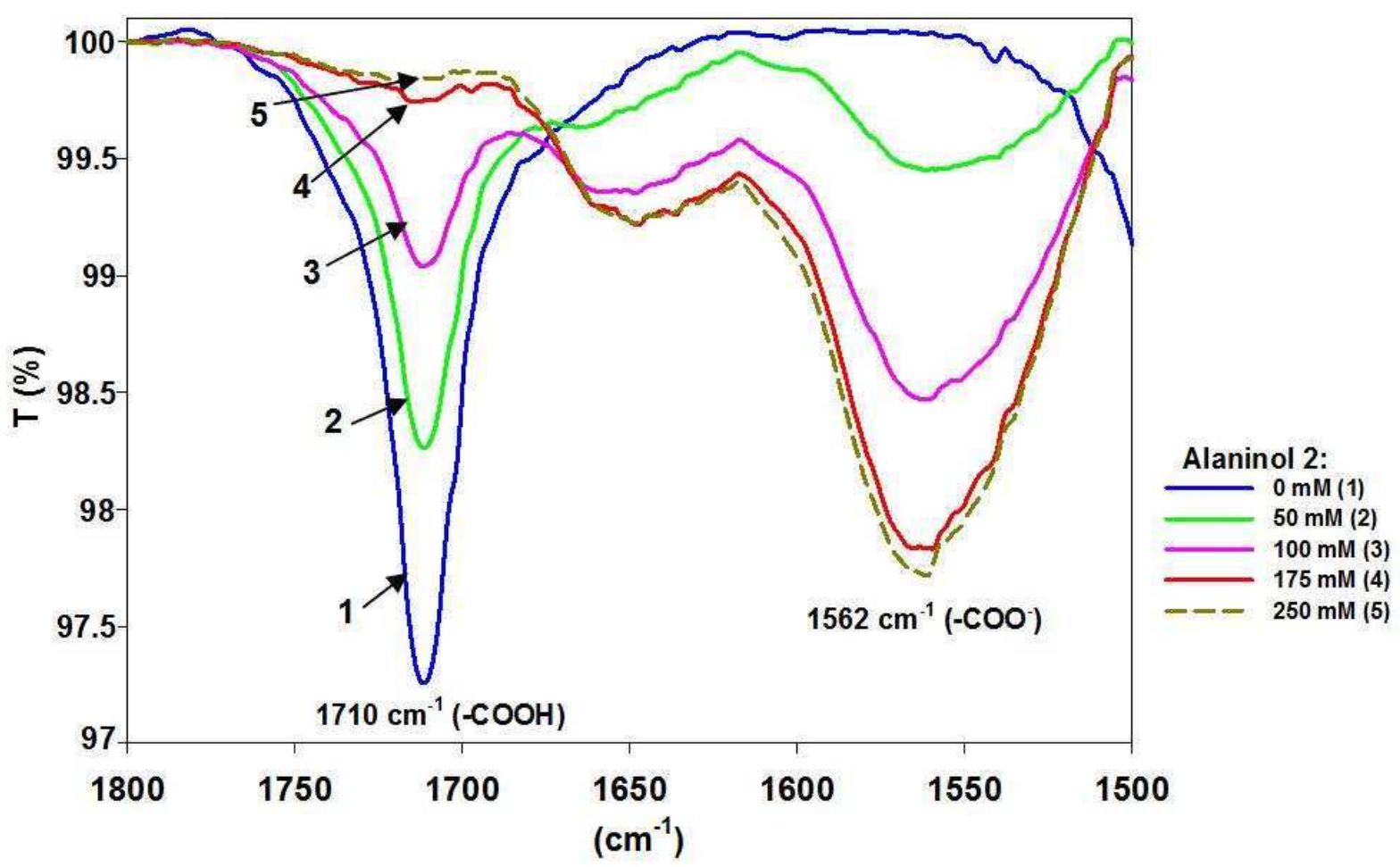

282 Fig. 2: IR analysis of mixtures containing $175 \mathrm{mM}$ of myristic acid and various 283 concentrations of alaninol in tert-amyl alcohol.

285 The kinetic studies resulting from the acylation of other acyl acceptors (Table 2) were 286 determined using the same method as the one used above for the acylation of alaninol. In all 287 kinetic profiles, a decrease in initial rates was observed for high concentrations of amino 288 substrates, most probably due to the formation of an ion-pair complex between substrates 289 similar to the complex described above in case of alaninol acylation. The apparent kinetic 290 parameters $K_{\text {m,app }}$ and $V_{\text {max,app }}$ resulting from the acylation of all acyl acceptors were 291 determined and are given on Table 3. 
Table 3. Apparent kinetics parameters for the N-acylation and O-acylation of various acyl acceptors using Candida antarctica lipase B in tert-amyl alcohol at $55^{\circ} \mathrm{C}$. Myristic acid (175

$300 \mathrm{mM}$ ) was used as an acyl donor.

\begin{tabular}{|c|c|c|c|c|}
\hline Entry & Acyl acceptor & $\begin{array}{l}K_{\mathrm{m}, \mathrm{app}} \\
(\mathrm{mM})\end{array}$ & $\begin{array}{c}V_{\text {max,app }} \text { of } \\
\text { N-acylation } \\
\left(m m o l \cdot h^{-1} \cdot \mathrm{g}^{-1}\right)\end{array}$ & $\begin{array}{c}V_{\text {max,app }} \text { of } \\
\text { O-acylation } \\
\left(\mathrm{mmol} \cdot \mathrm{h}^{-1} \cdot \mathrm{g}^{-1}\right)\end{array}$ \\
\hline 1 & $( \pm)$-alaninol 1 & 182 & 4.3 & - \\
\hline 2 & (R)-sec-butylamine 3 & 619 & 0.2 & - \\
\hline 3 & $\begin{array}{l}\text { (土)-1-methoxy- } \\
\text { 2-propylamine } 5\end{array}$ & 185 & 1.6 & - \\
\hline 4 & ( \pm -1,2-diaminopropane 7 & 252 & 7.6 & - \\
\hline 5 & $( \pm)$-4-amino-1-pentanol 9 & 75 & 1.1 & 7.3 \\
\hline 6 & 6-amino-1-hexanol 11 & 63 & 1 & 10.1 \\
\hline
\end{tabular}

301

302 Firstly, we compared the acylation resulting from a series of amines $(\mathbf{3}, \mathbf{5}$ and $\mathbf{7})$ structurally

303 related to amino-alcohol $\mathbf{1}$ (alaninol) with different groups in $\beta$ position of the amino group

304 (scheme 1 and Table 2, entries 1 to 4 ).

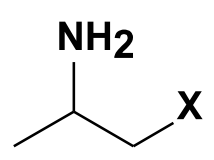

$$
\mathbf{X}=\mathrm{OH}(1), \mathrm{CH}_{3}(3), \mathrm{OCH}_{3}(5) \text { or } \mathrm{NH}_{2}(7)
$$

306 Scheme 1: Alaninol (1) and structurally related amines $(\mathbf{3}, \mathbf{5}, \mathbf{7})$.

307 The $K_{\mathrm{m} \text {,app }}$ of amine 3 ((R)-sec-butylamine) (619 mM; Table 3, entry 2) was 3-fold higher 308 than the $K_{\mathrm{m} \text {,app }}$ of amino-alcohol 1 (alaninol) (182 mM; Table 3, entry 1$)$. This showed a better 309 affinity of Candida antarctica lipase B toward a bifonctionnal amino-alcohol rather than a 310 monofunctional amine. On the other hand, the $K_{\text {m,app }}$ values of bifunctional compounds 1 
311 (alaninol), 5 (1-methoxy-2-propylamine) and 7 (1,2-diaminopropane), structurally related 312 substrates with two carbons between the two functional groups, were of the same order $\left(K_{\mathrm{m} \text {,app }}\right.$ 313 respectively equal to $182 \mathrm{mM}, 185 \mathrm{mM}$ and $252 \mathrm{mM}$; Table 3 , entries 1,3 and 4 ). On the

314 contrary, $V_{\text {max,app }}$ values of $\mathrm{N}$-acylation for compounds $\mathbf{1}, \mathbf{5}$ and $\mathbf{7}$ were variable (from 1.6 to $3157.6 \mathrm{mmol} \mathrm{h}^{-1} \mathrm{~g}^{-1}$ ), which showed that Candida antarctica lipase B catalytic efficiency was 316 mainly depending on its differential catalytic activity for these substrates. Furthermore, in a 317 previous work we have compared the acylation of mono-amine $\mathbf{3}((\mathrm{R})$-sec-butylamine) and a 318 structurally similar secondary alcohol: (R)-2-butanol. We noticed that the $V_{\max , a p p}$ of O319 acylation of (R)-2-butanol was 23-fold higher than the $V_{\max , a p p}$ of $\mathrm{N}$-acylation of amine 3 [20]. 320 These results were in contrast with those obtained for the acylation of alaninol, for which no 321 mono-O-acylation product was detected and a value of $V_{\text {max, app }}$ equal to $4.3 \mathrm{mmol} \mathrm{h}^{-1} \mathrm{~g}^{-1}$ was 322 obtained for $\mathrm{N}$-acylation. To better understand the influence of substrate structure on the 323 chemoselectivity of Candida antarctica lipase B, we compared the $V_{\max , a p p}$ of mono-N324 acylation of structurally related amines (scheme 1, compounds $\mathbf{1}, \mathbf{3}, \mathbf{5}$ and $\mathbf{7}$, Table 3, entries $3251,2,3$ and 4). The order of $V_{\max , a p p}$ values was found to be: diamine $7\left(7.6 \mathrm{mmol} \mathrm{h}^{-1} \mathrm{~g}^{-1}\right)>$ 326 amino-alcool $\mathbf{1}$ (alaninol) $\left(4.3 \mathrm{mmol} \mathrm{h}^{-1} \mathrm{~g}^{-1}\right)>$ methoxyamine $\mathbf{5}\left(1.6 \mathrm{mmol} \mathrm{h}^{-1} \mathrm{~g}^{-1}\right)>>$ amine $\mathbf{3}$ $327\left(0.2\right.$ mmole $\left.^{-1} \mathrm{~g}^{-1}\right)$.The first substrate diamine 7, which exhibits the highest $V_{\text {max,app }}$ values 328 was mono-N-acylated only in position 1. Indeed, only mono-amide 8a was detected (Table 2, 329 entry 4). The last substrate amine 3 is a monofunctional amine with no nucleophilic group in $330 \quad \beta$-position of an amino group. From these results, we could thus conclude that the presence of 331 a nucleophilic group (alcohol group, methoxy group or a second amino group) in $\beta$-position 332 of the acyl-acceptor amino group was responsible for the enhancement of $V_{\text {max,app }}$ of $\mathrm{N}$ 333 acylation of this amino group and that the more nucleophilic group $\left(-\mathrm{NH}_{2}>-\mathrm{OH}>-\mathrm{OCH}_{3}\right)$ in $334 \beta$-position, the higher the reactivity for the amino group. 
335 Secondly, we compared the acylation of three amino-alcohols that exhibited a variable carbon 336 chain length between the amino and alcohol groups: amino-alcohols 1 (two carbons), 9 (four carbons) and 11 (six carbons) (Table 2, entries 1, 5 and 6). In terms of $K_{\mathrm{m} \text {,app }}$ values, the $K_{\mathrm{m} \text {,app }}$ 338 of amino-alcohol 9 (75 mM; Table 3, entry 5) was in the same order than the $K_{\mathrm{m} \text {,app }}$ of amino339 alcohol 11 (63 mM; Table 3, entry 6), whereas the $K_{\text {m,app }}$ of amino-alcohol 1 (182 mM; Table 340 3, entry 1) was higher. This pointed out a better affinity of Candida antarctica lipase B 341 toward long chain amino-alcohols 9 and $\mathbf{1 1}$ than for short amino-alcohols $\mathbf{1}$. On the other 342 hand, the acylation of long chain amino-alcohols 9 and $\mathbf{1 1}$ by Candida antarctica lipase B 343 gave $V_{\text {max,app }}$ of $\mathrm{N}$-acylation of 1.1 and $1 \mathrm{mmol} \mathrm{h}^{-1} \mathrm{~g}^{-1}$ (Table 3, entries 5 and 6), respectively, 344 as short chain amino-alcohol 1 was $\mathrm{N}$-acylated 4-fold faster ( $V_{\text {max,app }}$ of $\mathrm{N}$-acylation: $4.3 \mathrm{mmol}$ $345 \mathrm{~h}^{-1} \mathrm{~g}^{-1}$; Table 3, entry 1) than long chain amino-alcohols. In contrast, $V_{\text {max,app }}$ of O-acylation of $3467.3 \mathrm{mmol} \mathrm{h}^{-1} \mathrm{~g}^{-1}$ for amino-alcohol 9 and $10.1 \mathrm{mmol} \mathrm{h}^{-1} \mathrm{~g}^{-1}$ for amino-alcohol 11 (Table 3, 347 entries 5 and 6) were obtained, whereas no mono-O-acylated product and only trace amounts 348 of amido-ester $2 \mathbf{c}$ were detected during the acylation of short chain amino-alcohol $\mathbf{1}$. This was 349 attributed to the fact that the reaction could take place at the amino group (N-acylation) and/or 350 alcohol group (O-acylation) of long chain amino-alcohols 9 and 11, giving either $\mathrm{N}$ - or O351 acylated products 10a, 10b 12a and 12b (Table 2, entries 5 and 6), while the mono-O352 acylation of short chain amino-alcohol 1 did not occur. Starting from these results, we could 353 calculate the chemoselectivity ratio (Eq. (2)) of the Candida antarctica lipase B-catalyzed 354 acylation of long chain amino-alcohols 9 and 11, which was close to 6.6 and 10.1, 355 respectively. From these results, we could conclude that the increase in the carbon chain 356 length between the alcohol and amino groups of long chain amino-alcohols was concomitant 357 with the increase in the chemoselectivity of Candida antarctica lipase B for the O-acylation 358 of these substrates. 
To interpret all these data resulting from the acylation of structurally related amines $(\mathbf{1}, \mathbf{3}, \mathbf{5}$

360 and 7) and amino-alcohols with various carbon chain length (1, 9 and $\mathbf{1 1})$, we formulated the 361 following postulate: the presence of a nucleophilic group in $\beta$-position of the amino group of

362 the acyl acceptor amine resulted in the enhancement of the $V_{\text {max,app }}$ of $\mathrm{N}$-acylation of this 363 amino group. This may be due to the formation of an intramolecular interaction between the 364 amino group and the nucleophilic group located in $\beta$-position, which is strengthened by the 365 fact that this interaction could not occur for long chain amino-alcohols 9 and 11, considering 366 the longer distance between both functional groups, giving as a result a decrease in the $V_{\text {max,app }}$ 367 of N-acylation.

\section{Conclusion}

369 In this work, we investigated the Candida antarctica lipase B-catalyzed acylation of various 370 amines and amino-alcohols as acyl acceptors, using myristic acid as an acyl donor, and 371 showed that the presence of a nucleophilic group $\left(-\mathrm{NH}_{2}\right.$ or $-\mathrm{OH}$ or $\left.-\mathrm{OCH}_{3}\right)$ in $\beta$-position of the 372 amino group of the acyl acceptor enhances the $V_{\max , a p p}$ of $\mathrm{N}$-acylation and thus the enzyme 373 activity. Moreover, the crucial role of the carbon chain length between the alcohol and amino 374 groups was highlighted in the Candida antarctica lipase B-catalyzed acylation of amino375 alcohols. The $V_{\text {max,app }}$ of $\mathrm{N}$-acylation was indeed improved when the carbon chain included 376 two carbons (alaninol 1) whereas the $V_{\text {max,app }}$ of O-acylation was improved when the carbon 377 chain included four carbons or more (4-amino-1-pentanol 9 and 6-amino-1-hexanol 11). The 378 present investigation demonstrated the great influence of substrate structure on the 379 chemoselectivity of Candida antarctica lipase B, providing new insights for the selective 380 synthesis of amides or esters produced from the acylation of bifunctional substrates. 381 Molecular modelling studies are currently in progress to study in details the acylation 382 mechanism of amino-alcohols. 
383 Finally, the ability to understand and control the chemoselectivity of Candida antarctica 384 lipase B, apart from its interest in the specific acylation of bifunctional substrates, constitutes 385 a promising enzymatic way to acylate other heterofunctional compounds such as precursors of 386 ceramide synthesis (eg sphingoid bases or other amino-polyols) or precursors of amino-acid 387 based surfactant synthesis (eg amino-acids or peptides). 
400 Acknowledgments

401 This study was supported by the Centre National de la Recherche Scientifique and the French 402 ANR (National Research Agency) through the EXPENANTIO project (CP2P program: 403 Chimie et Procédés pour le Développement Durable).

404 


\section{References}

406 [1] M. Bakke, M. Takizawa, T. Sugai, H. Ohta, J. Org. Chem., 1998, 63, 6929-6938.

407 [2] L. Lassalle, F. Yvergnaux. FR Patent 2,855,823 (2004).

408 [3] H. Garg, N. Francella, K.A. Tony, L.A. Augustine, J.J. Barchi Jr, J. Fantini, A. Puri, 409 D.R. Mootoo, R. Blumenthal. Antiviral Res., 2008, 80, 54-61.

410 [4] M. Fillet, M. Bentires-Alj, V. Deregowski, R. Greimers, J. Gielen, J. Piette, V. Bours, 411 M.P. Merville, Biochem Pharmacol., 2003, 65, 1633-1642.

412 [5] J.F. Molina, Household and personal care today. 2 (2008) 12-15.

413 [6] E.L. Soo, A.B. Salleh, M. Basri, R.N.Z.R.A. Rahman, K. Kamaruddin, J. Biosci. 414 Bioeng., 2003, 95, 361-367.

415 [7] A. Singh, J.D. Van Hamme, O.P. Ward, Biotech Adv., 2007, 25, 99-121.

416 [8] T. Maugard, M. Remaud-Simeon, D. Petre, P. Monsan, Tetrahedron, 1997, 53, 51854175194.

418 [9] M. Fernandez-Perez, C. Otero, Enzyme Microb. Technol., 2003, 33 (5), 650-660.

419 [10] M. Fernandez-Perez, C. Otero, Enzyme Microb. Technol., 2001, 28 (6), 527-536.

420 [11] V. Gotor, R. Brieva, F. Rebolledo, J. Chem. Soc., Chem. Commun., 1988, 957-958.

421 [12] T. Furutani, M. Furui, H. Ooshima, J. Kato, Enzyme Microb. Technol., 1996, 19 (8), $422 \quad 578-584$.

423 [13] L.T. Kanerva, E. V“anttinen, T.T. Huuhtanen, M. Dahlqvist, Acta chem. Scand, 1992, $42446(11), 1101-1105$.

425 [14] O. Torre, V. Gotor-Fernandez, V. Gotor, Tetrahedron Asymmetry, 2006, 17 (5), 860426866. 
427 [15] S. Tawaki, A.M. Klibanov, Biocatal. Biotransform., 1993, 8, 3-19.

428 [16] T. Maugard, M. Remaud-Simeon, D. Petre, P. Monsan, Tetrahedron., 1997, 53, 75874297594.

430 [17] A. Fersht, Enzyme Structure and Mechanism, second ed., W. H. Freeman and 431 Company, New York, 1985, pp. 98-120.

432 [18] E. Husson, C. Humeau, F. Blanchard, X. Framboisier, I. Marc, I. Chevalot, J. Mol. 433 Catal. B: Enzym., 2008, 55, 110-117.

434 [19] M. Fernandez-Perez, C. Otero, Enzyme Microb. Technol., 2001, 28, 527-536.

435 [20] F. Le Joubioux, O. Achour, N. Bridiau, M. Graber, T. Maugard, J. Mol. Catal. B: 436 Enzym., 2011, 70, 108-113. 